\title{
MELANOMA: PERFIL EPIDEMIOLÓGICO DE CINCO ANOS EM UM HOSPITAL DE CURITIBA-PR
}

\section{MELANOMA: EPIDEMIOLOGICAL PROFILE OF FIVE YEARS IN A HOSPITAL OF CURITIBA-PR}

DOI: $10.5380 /$ rmu.v2i2.40993

Valéria Aparecida Zanela Franzon ${ }^{1}$, Luiara Stefanelo Loro², Gustavo Pandolfo²

\section{RESUMO}

Introdução: O melanoma cutâneo (MC) é a terceira neoplasia mais frequente na pele, entretanto é a de pior prognóstico. Mesmo sendo pouco prevalente, representa uma séria ameaça de saúde pública em virtude de ocasionar altas taxas de morbimortalidade. Objetivo: $\mathrm{O}$ objetivo deste estudo é realizar uma triagem nos prontuários de pacientes diagnosticados com $\mathrm{MC}$ durante cinco anos em um serviço especializado em câncer de pele da cidade de Curitiba/PR, a fim de conhecer as características desta neoplasia nessa população. Métodos: Realizou-se uma análise restrospectiva através da verificação de prontuários de pacientes com MC, diagnosticados no período compreendido entre janeiro de 2007 e dezembro de 2011, em um serviço especializado em câncer de pele da cidade de Curitiba/PR. Resultados: A amostra consistiu em 23 casos de MC de sítio primário, sendo lentigo maligno (43,4\%), extensivo superficial (26\%), nodular $(17,6 \%$ ) e não especificado (13\%). Dentre estes 3 com Breslow $\geq 4 \mathrm{~mm}$, todos em pacientes do sexo masculino. Em contrapartida, em $43,4 \%$ dos pacientes não foi possível aplicar o índice de Breslow, em virtude do lentigo maligno melanoma caracterizar-se por ser in situ. Identificou-se homens com diagnóstico mais tardio em relação às mulheres, já que $80 \%$ deles possuíam um Breslow $>1 \mathrm{~mm}$. Conclusão: Sugere-se maior divulgação dos cuidados com a exposição solar e melhor formação para que o médico clínico geral seja um identificador de MC o mais precocemente possível, podendo, assim, encaminhar o paciente para uma abordagem com maior possibilidade de cura.

Palavras-Chave: Epidemiologia; Diagnóstico; Melanoma.

\section{ABSTRACT}

Introduction: The cutaneous melanoma (CM) is the third most common skin cancer, however, has the worst prognosis. Even being little prevalent, is a serious worring due to high morbidity and mortality rates. Objective: To perform a screening in the medical records of MC diagnoses for five years in a skin cancer specialized center in Curitiba / PR, with the view to know the characteristics of this cancer in this population. Methods: A retrospective analysis was performed by checking records of patients with MC, diagnosed in the period between January 2007 and December 2011, in a skin cancer specialized center in Curitiba / PR. Results: 23 patients were selected, and lentigo malign (43.4\%), superficial spreading (26\%), nodular (17.6\%) and unspecified (13\%). Among these 3 with Breslow $\geq 4 \mathrm{~mm}$, all in male patients. However, in $43.4 \%$ of patients was not possible to apply the Breslow thickness, due to lentigo malign melanoma characterized by being in situ. Men were diagnosed later in relation to women, since $80 \%$ of them had a Breslow> $1 \mathrm{~mm}$. Conclusion: It is suggested wider disclosure of sun protection and better training for the primary care physician, and thus can route the patient to an approach with greater chances of cure.

Key words: Epidemiology; diagnosis; Melanoma.

\author{
1-Professora do Curso de Medicina da Pontifícia Universidade Católica do Paraná - PUCPR e Dermatologista associada \\ da Sociedade Brasileira de Dermatologia \\ 2- Aluno do Curso de Medicina da Pontifícia Universidade Católica do Paraná - PUCPR
}

Luiara Stefanelo Loro - luiara_sloro@hotmail.com

Rua Imaculada Conceição, 1155 - Bairro Prado Velho - CEP: 80215-901 
INTRODUÇÃO

O melanoma cutâneo (MC) é considerado o mais maligno dos tumores de pele. ${ }^{1,2}$ Origina-se a partir da transformação maligna dos melanócitos. ${ }^{3-6} \mathrm{~A}$ quantidade dessas células é similar entre brancos e negros, no entanto, a fotoproteção é prejudicada em pessoas de pele branca devido a menor produção de melanina. Sendo assim, a prevalência de MCé maior em indivíduos com fototipos menores. $\mathrm{E}$ mais incidente, também, no sexo masculino entre os 30 e 60 anos de idade. $^{\text {7-11 }}$

No âmbito mundial, a incidência anual do MC é cerca de 4 a $8 \%^{3}$. Ele representa ainda aproximadamente $5 \%$ dos casos novos de neoplasias no mundo. ${ }^{4}$ No Brasil, o câncer de pele é considerado o mais prevalente dentre todas as formas de câncer (25\%) e o MC representa $4 \%$ destes. $^{7}$

Vários fatores de risco têm sido associados a gênese de melanomas cutâneos, sendo os principais: genética, exposição solar e fenótipo. ${ }^{3,4} \mathrm{~A}$ genética é o fator de risco mais influente no desenvolvimento de MC. ${ }^{1,5}$ Indivíduos com histórico familiar da doença possuem maior probabilidade de desenvolvê-la. ${ }^{4} \mathrm{O}$ tumor nestes pacientes se desenvolve por volta dos 40 anos, e se caracteriza por múltiplas lesões primárias e nevos displásicos, sendo essas as características da Síndrome dos Nevos Displásicos. ${ }^{4,5}$

A exposição solar contribui para o surgimento do MC em indivíduos de pele clara e algumas evidências embasam esta afirmação: ${ }^{5,12}$ 1) melanomas cutâneos se localizam predominantemente em áreas do corpo mais expostas ao sol, como ombros e pernas; ${ }^{3,4,12}$ 2) a incidência e a mortalidade do MCsão diretamente proporcionais a intensidade de sol de uma determinada região,ou seja, as menores latitudes recebem uma maior intensidade de radiação solar e a incidência de $M C$ nesses territórios também é maior; ; 3 ) a incidência de $M C$ em brancos é maior do que em negros moradores de um mesmo localem virtude dos negros possuírem uma maior quantidade de melanina na pele e uma menor propensão a nevos. ${ }^{1,3,4,7,8,10}$

O fenótipo que compreende olhos claros, pele clara, cabelos loiros ou ruivos, presença de nevos na pele, tem maior tendência a desenvolver MC. ${ }^{1-5,11,13-16}$

O MC primário pode ser classificado em: lentigo maligno melanoma, melanoma extensivo superficial, melanoma nodular, melanoma lentiginoso acral de acordo com as características clínicas e patológicas. ${ }^{3,4,13,14} \mathrm{O}$ exame anatomopatológico de um MC deve incluir ainda o índice deBreslow, definido como a relação da profundidade em que as células tumorais invadiram a pele com a sobrevida do paciente em 10 anos (Tabela 1). ${ }^{17}$ A American Joint CommitteeonCancer (AJCC) validou a importância da profundidade do tumor como o mais importante fator prognóstico em MC, seguido de ulceração histopatologicamente reconhecida e a identificação de micro metástases nos linfonodos regionais através da técnica da biópsia do linfonodo sentinela. ${ }^{6,13,18}$

Tabela 01 - Índice De Breslow

\begin{tabular}{ll}
\hline Profundidade & Sobrevida (10a) \\
\hline$<1 \mathrm{~mm}$ & $91 \%$ \\
$1,1-2 \mathrm{~mm}$ & $74 \%$ \\
$2,1-4 \mathrm{~mm}$ & $57 \%$ \\
$>4 \mathrm{~mm}$ & $44 \%$ \\
\hline
\end{tabular}

Fonte: Adaptado de: Sampaio SA, Rivitti EA.(2008) $)^{3}$

Os níveis de Clark descrevem a invasão anatômica do $\mathrm{MC}$ na pele, como pode ser observado na tabela $2 .{ }^{17}$ Segundo o AJCC esta classificação não é mais recomendada como critério de estadiamento. ${ }^{18}$ Porém, é recomendado pela Diretriz Brasileira a presença do nível de Clark no laudo anatomopatológico para fins de estadiamento. ${ }^{19}$

\section{Tabela 02 - Níveis de Clark}

\begin{tabular}{ll}
\hline Nível & \\
\hline $\mathbf{1}$ & Confinado a epiderme (in situ) \\
$\mathbf{2}$ & Invasão da derme papilar \\
$\mathbf{3}$ & Invasão da junção derme papilar-reticular \\
$\mathbf{5}$ & Invasão da derme reticular \\
\hline
\end{tabular}

Fonte: Ministério da Saúde $(2013)^{20}$

O objetivo do presente artigo é a revisão de prontuários de um serviço de dermatologia no intervalo de cinco anos para identificar o perfil epidemiológico do MC em pacientes atendidos pelo Sistema Único de Saúde (SUS), apresentando dados estatísticos que poderão servir de referência para estudos epidemiológicos e prevenção no Sul do Brasil.

\section{METODOLOGIA}

Realizou-se uma análise retrospectiva através do levantamento de 561 prontuários de pacientes submetidos à biópsia de pele em um serviço especializado em câncer de pele da cidade de Curitiba/PR, no período compreendido entre janeiro de 2007 e dezembro de 2011. Vinte e três casos de MC foram encotrados. 


\section{REVISTA MÉDICA DA UFPR \\ ISSN: 2358-193X}

Foram avaliadas características clínicas da lesão em primeira consulta (simetria, cor, diâmetro, localização da lesão e aspectos dermatoscópicos). Foram valorizados ainda para fins de estudo epidemiológico: sexo, idade e cidade de origem. E analisou-se, também, o laudo do exame anatomopatológico e a morfologia do MC (estágio de invasão usando os níveis de Breslow e de Clark).

\section{RESULTADOS}

A amostra total foi composta por 23 pacientes portadores de MC (Gráfico 1): 9 (39,13\%) apresentavam lentigo maligno melanoma, $6(26,08 \%)$ melanoma extensivo superficial e $5(21,73 \%)$ melanoma nodular. No exame anatomopatológico de $3(13,0 \%)$ pacientes não foi especificado o tipo do MC.

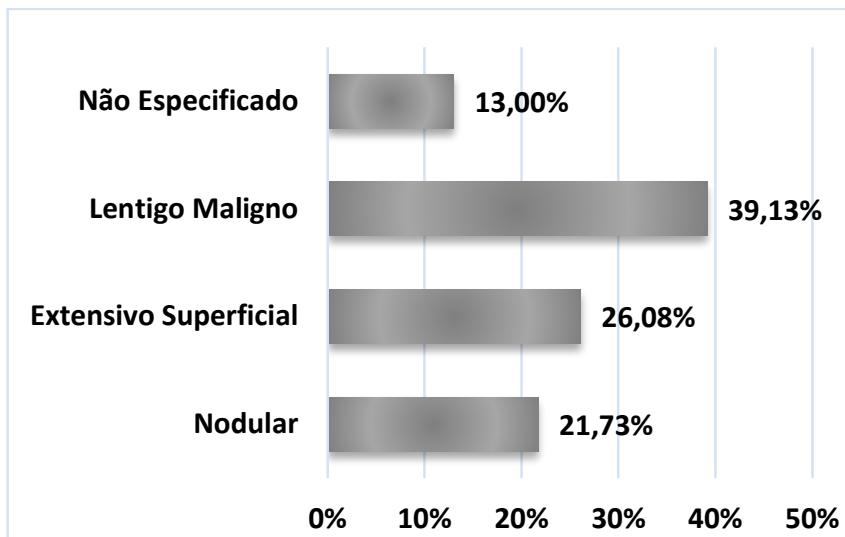

Gráfico 1 - Tipos histológicos de MC encontrados nos prontuários.

Quanto as características epidemiológicas, o grupo de MC foi composto por 14 (60,9\%) mulheres e 9 $(39,1 \%)$ homens, como pode-se evidenciar no gráfico 2 . Desse modo, é possível determinar uma relação da presença de $M C$ entre os sexos, sendo esta de 1,5 mulheres para cada homem. Ainda a respeito dos sexos, observou-se em 3 pacientes a presença de MC com índice de Breslow $>4 \mathrm{~mm}$, e desses todos eram do sexo masculino. Assim sendo, $80 \%$ dos homens foram diagnosticados com Breslow $>1 \mathrm{~mm}$, enquanto, entre as mulheres 70,4\% diagnosticaram o câncer com Breslow $\leq 1 \mathrm{~mm}$. Em relação as faixas etárias, a idade média da amostra consistiu em 63,6 anos, devido a maior parte dos pacientes apresentarem-se com idade superior a 50 anos (91,3\%). Apenas $34,8 \%$ dos pacientes residiam na cidade de Curitiba.

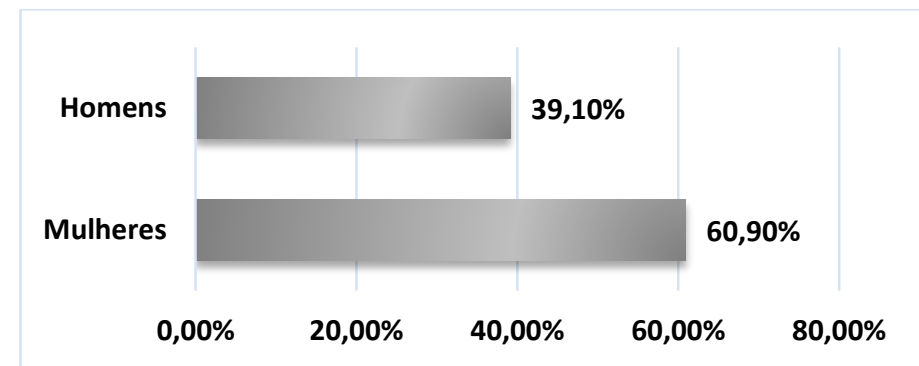

Gráfico 2: Relação do MC quanto ao sexo

Quanto ao Índice de Breslow, 3 (13\%) pacientes apresentaram profundidade da lesão igual ou maior que $4,0 \mathrm{~mm}$.

Conforme à topografia, as lesões situaram-se nos membros superiores ( $n=3 ; 13,1 \%$ ), nos membros inferiores ( $n=2 ; 8,7 \%$ ), no tronco $(n=9 ; 39,1 \%)$, na região da cabeça e pescoço ( $n=8 ; 34,8 \%$ ), e não especificada a localização no prontuário $(n=1 ; 4,3 \%)$. As mulheres apresentaram tumores predominantemente na região da cabeça e pescoço $(30,8 \%)$ e no tronco $(30,8 \%)$. Bem como, os homens possuíam tumores principalmente no tronco $(55,5 \%)$ e na região da cabeça e pescoço $(44,5 \%)$.

\section{DISCUSSÃO}

A proposta do presente estudo é apresentar o perfil epidemiológico do $M C$ de cinco anos de atendimento de um hospital universitário na cidade de Curitiba-PR. No entanto, sabendo do aumento progressivo da incidência do $\mathrm{MC}$ em âmbito mundial, questionou-se a respeito da pequena amostra encontrada. A única hipótese plausível considerada levou em conta a demora de cerca de 4 meses para o acesso a um dermatologista pelo SUS. Diante dessa lentidão, os médicos da atenção primária estão encaminhando os pacientes portadores delesões suspeitas diretamente ao serviço de referência em oncologia no estado do Paraná, o qual atende os pacientes em, no máximo, 15 dias após a solicitação da consulta. Apesar dessa limitação, observou-se um predomínio de MC do tipolentigo maligno melanoma, justificado pela faixa etária predominantemente idosa investigada, ${ }^{1,3,4}$ seguido de extensivo superficial e nodular. Dados estes que contrariam as estatísticas de um estudo de Porto Alegre, o qual apresentou $62,8 \%$ do tipo extensivo superficial. ${ }^{13}$

Em relação a topografia, encontrou-se no sexo feminino a presença de MC principalmente na parte superior do corpo, dado este justificado novamente pelo tipo histológico lentigo maligno melanoma, o qual predomina em áreas expostas. 3,4,11-13,21-24 Houve também concordância com as revisões no sexo masculino, em que os tumores localizaram-se especialmente em região de cabeça e pescoço - nesta 
amostra - maioria no tronco $(55 \%)^{3,4,6,11,12,13,15,21-24}$. Apesar da amostra não conferir significância estatística quanto a topografia, os dados encontrados coincidem com a literatura, sendo: lentigo maligno melanoma predominante em cabeça e pescoço; extensivo superficial e nodular em tronco e membros, respectivamente ${ }^{3,4,11,12,13,21-23}$.

Assim como refere a literatura, notou-se nesse estudo uma discreta incidência maior de $M C$ no sexo feminino. ${ }^{6,11,17}$ Entretanto, a avaliação de prevalência quanto ao sexo não mostrou relevância estatística nesta análise. Identificou-se também que os homens recebem um diagnóstico mais tardio em relação às mulheres, e atribuí-se isto à maior preocupação delas quanto a saúde ${ }^{25}$.

Em relação a distribuição dos subtipos histológicos, a presente pesquisa evidenciou uma amostra composta por $39,13 \%$ de MC do tipo lentigo maligno melanoma, o que difere de muitos achados na literatura. ${ }^{1,3,4,8,11,13,17,26,27}$ Estudos de base hospitalar das décadas de 90 e 2000 indicam predomínio do subtipo extensivo superficial. ${ }^{11,13}$ Esta diferença pode estar relacionada, em parte, a amostra do presente estudo ser composta de pacientes na faixa etária média de 65 anos, e entre idosos, o lentigo maligno melanoma é o mais prevalente, segundo a literatura. , $3,4,11^{-1}$

$\mathrm{O} \mathrm{MC}$ mesmo sendo pouco prevalente entre os tumores malignos de pele (4\%) é uma séria ameaça de saúde pública. ${ }^{17,16}$ Isso porque é um câncer com uma alta taxa de morbimortalidade. ${ }^{2,5,16,17} \mathrm{O}$ melhor meio de combatê-lo é o diagnóstico precoce, pois ao diagnosticar um $\mathrm{MC}$ com Breslow $<1 \mathrm{~mm}$ a taxa de sobrevida chega a $92 \%{ }^{1,18} \mathrm{Em}$ contrapartida, o diagnóstico tardio - Breslow $\geq 4 \mathrm{~mm}$ - confere uma sobrevida de apenas $44 \%$ em 10 anos. 4,5,18,28,29

Há três níveis de prevenção para a identificação dos estágios iniciais de câncer: a primária, a secundária e a terciária. A primária previne a ocorrência da enfermidade, ${ }^{24}$ porém ela nem sempre é possível nos casos familiares.A secundária consiste no diagnóstico precoce por meio de rastreamento e exérese de lesões pigmentadas com sinais de atipia. ${ }^{24}$ Nesse tipo de prevenção, o uso do $A B C D E$ ( $A$ de assimetria, $B$ de bordas irregulares, $C$ de cores heterogêneas, $D$ de diâmetro superior a $6 \mathrm{~mm}$ e $E$ de evolução durante $o$ tempo $)^{3,4}$ se torna uma ferramenta diagnósticade grande valia ao clínico na Unidade Básica de Saúde a fim de encaminhar precocemente o paciente com lesão suspeita ao serviço especializado. Já a terciária prevê o acompanhamento de lesões inoperáveis e a instituição de terapêuticas muitas vezes experimentais. ${ }^{24}$ Sendo assim, os níveis primário e secundário são os mais importantes para uma neoplasia com alta incidência de mortalidade como é o MC, devendo ser o foco das campanhas públicas. ${ }^{30}$

O foco principal da prevenção primária contra o câncer de pele é a proteção solar, pois acredita-se que a radiação solar, particularmente a ultravioleta, provoque danos diretos ao DNA., ${ }^{51}$ Portanto, sugere-se campanhas públicas para retificar a importância de se proteger contra o sol. Enfatizando a proteção mecânica - uso de chapéus, bonés, óculos-de-sol - e a proteção química com o uso dos filtros solares.

O enfoque da prevenção secundária é o diagnóstico precoce. Para isso, é necessário orientar a população a respeito das características malignas de uma lesão pigmentada. ${ }^{5,16,24,31}$ Sugere-se tornar a população uma grande massa de identificadores de lesões suspeitas, afinal a precocidade do diagnóstico do MC interfere diretamente na sobrevida do paciente pa,13,17,21. $^{3,}$.

A limitação do estudo foi um pequeno número de casos, apesar da revisão de prontuários de cinco anos. Além disso, alguns dados importantes dos prontuários foram negligenciados no decorrer dos atendimentos. Sugerimos, então, um cuidado especial no registro dos pacientes com tumores de pele. É de suma importância a descrição de informações básicas a respeito do tumor, como: tamanho, localização, aspecto, textura, cor e evolução da lesão a cada nova consulta ${ }^{3,4,20}$. Realização de dermatoscopia, bem como a sua descrição ${ }^{20}$. O registro das biópsias com data e laudo também é necessário. O laudo descritivo, que no caso do MC inclui profundidade de Breslow, ulceração, invasão perineural, micro metástases, níveis de Clark e as margens da lesão, deveriam ser inteiramente transcritos no prontuário do paciente ${ }^{20}$.

\section{CONCLUSÃO}

O perfil dos casos de MC avaliados em um hospital terciário de referência de Curitiba apresentou um viés de faixa etária. Porém, tendo em vista que a população da região Sul brasileira, pelas condições de colonização, apresenta um grupo representativo de fototipos I e II de Fitzpatrick, sugerindo maior divulgação dos cuidados com a exposição solar e melhor formação para que o médico clínico geral seja um identificador de MC o mais precocemente possível, podendo, assim, encaminhar o paciente para uma abordagem com maior possibilidade de cura.

O médico de atenção primária esteja preparado e atento para suspeitar de lesões pigmentares atípicas, encaminhando-as o mais breve para o atendimento especializado. Além de fazer-se necessário o treinamento dos profissionais de saúde e esclarecimento populacional quanto a esta doença.

\section{REFERÊNCIAS}

1. Dimatos DC, Duarte FO, Vieira VJ, Vasconcellos ZA, Bins-Ely J, et al. Melanoma cutâneo no Brasil. Arq Cat de Medicina. 2009; 38:14-9. 


\section{REVISTA MÉDICA DA UFPR}

ISSN: 2358-193X

2. Jung JE, Júnior RA, Gennaro L, Leme FE, Martins AP, Hirth $C G$, et al. Análise imuno-histoquímica dos marcadores de progressão tumoral, E-caderina, $\beta$-catenina, CEACAM-1 e PTEN em melanomas. J Bras Patol Med Lab. Abril 2010; 46:111-8.

3. Sampaio SA, Rivitti EA. Dermatologia. 3.ed. São Paulo, Brasil: Artes Médicas Editora; 2008.

4. Freedberg IM, Eisen AZ, Wolff K, Austen KF, Goldsmith LA, Katz, S.I., Fitzpatrick, T.B. Fitzpatrick's dermatology in General Medicine. 8th ed. New York: The McGraw-Hill Co.; 2012.

5. Zaidi MR, Day C, Merlino G. From UVs to metastases: modeling melanoma initiation and progression in the mouse. J of Invest Dermat. 2008; 128: 2381-91.

6. Botella-Estrada $R$, Jiménez $S$. Diferentes alteraciones genéticas sausan diferentes melanomas y nuevas possibilidades terapêuticas. Actas Dermosifiliogr [internet]. 2010 [acesso em 2013 set 26]; 101: 394-400. Disponível em: http://http://www.actasdermo.org

7. INCA (Instituto Nacional do Câncer. - Ministério da Saúde. Estimativas da Incidência e Mortalidade por Câncer. Disponível em: <http://www.inca.gov.br> Acesso jun. 2014.

8. Herrerias T. Efeitos de flavonóides sobre o metabolismo mitocondrial e suas implicações na viabilidade e apoptose de células de melanoma [dissertação de doutorado]. Curitiba: Universidade Federal do Paraná; 2009.

9. Herlyn M, Satyamoorthy K. Efeitos de flavonóides sobre o metabolismo mitocondrial e suas implicações na viabilidade e apoptose de células de melanoma [dissertação de doutorado]. Curitiba: Universidade Federal do Paraná; 2009.

10. Alchorne MM, de Abreu MA. Dermatologia na pele negra. An Bras Dermatol. 2008; 83:7-20.

11. Maia M, Ferrari N, Russo C, Ribeiro MC, Santos AB. Reflexões em relação à epidemiologia do melanoma cutâneo no Brasil. An Bras Dermatol. 2002; 77:163-70.

12. Vilanova CM, Lages RB, Ribeiro SM, Almeida IP, Santos LG, Vieira SC. Perfil epidemiológico e histopatológico do melanoma cutâneo em um centro do nordeste brasileiro de 2000 a 2010. An Bras Dermatol. 2013;88:553-62.

13. Bonfá R, Bonfá R, Furian RD, Bonamigo RR, Duro KM, Zelmanowicz AM. A precocidade diagnóstica do melanoma cutâneo: uma observação no sul do Brasil. An Bras Dermatol. 2011;86:215-21.

14. Tincani AJ, Negro AD. Diagnóstico e tratamento do melanoma cutâneo. Depto. Cirurgia de Cabeça e Pescoço - FCM Unicamp.

15. Naser N. Melanoma cutâneo - estudo epidemiológico de 30 anos em cidade do Sul do Brasil, de 1980-2009. An Bras Dermatol. 2011;86:932-41.

16. Salvio AG, Segalla JG, Nicolini HR, Júnior AA, Panfilo BL, Didone R. Experiência de um ano de modelo de programa de prevenção contínua do melanoma na cidade de Jaú-SP, Brasil. An Bras Dermatol. 2011; 86:669-74.

17. Proença NG, Bernardes MF, Muller $H$, Jorge D, Frucchi $H$, Boix AS, et al. Fatores que influenciam sobre o prognóstico do melanoma maligno. An Bras Dermatol. 1991; 66:19-24.

18. Balch CM, Gershenwald JE, Soong S, Thompson JF, Atkins MB, Byrd DR, et al. Final version of 2009 AJCC melanoma staging and classification. J of Clinic Oncol [internet]. 2009 [acesso em 2014 jun 18]; 27(36.:6199-6206. Disponível em: http:www.jco.ascopubs.org 19. Tovo FL, Belfort FA, Sanches JA. Melanoma cutâneo abordagem da lesão primária. Projeto Diretrizes. Socie Bras de Dermatol. 2002.

20. Brasil. Ministério da Saúde. Portaria no 357, de 08 de abril de 2013. Diretrizes diagnósticas e terapêuticas do melanoma maligno cutâneo [para diagnóstico, tratamento e acompanhamento dos indivíduos com melanoma cutâneo]. Diário Oficial da República Federativa do Brasil. 2013 abril 08; Seção 1.p.48-52.

21. Konrad P, Fabris MR, Melao S, Blanco LFO. Perfil epidemiológico e histopatológico dos casos de melanoma cutâneo primário diagnosticados em Criciúma no período entre 2005 e 2007. An Bras Dermatol. 2011;86(3):457-61.
22. Junior NM, Ferrari J, et al. Melanoma cutâneo: estudo epidemiológico descritivo. Sao Paulo Med J [internet]. 2008 [acesso em 2013 ago];126:41-7. Disponível em:http://dx.doi.org/10.1590

23. Lipsker D, Engel F, Cribier B, Velten M, Hedelin G. Trends in melanoma epidemiology suggest three different types of melanoma. Br J Dermatol. 2007;157:338-43.

24. Maia M, Basso M. Quem descobre o melanoma cutâneo. An Bras Dermatol. 2006;81(3):244-8.

25. Diogo MJ, Ceolim MF, Cintra FA. Orientações para idosas que cuidam de idosos no domicílio. Rev Esc Enferm USP. 2005; 39(1):97-102.

26. Weber AL, Nunes DH, Souza Filho JJ, Pinto CJC. Avaliação de 496 laudos anatomopatológicos de melanoma diagnosticados no município de Florianópolis, Santa Catarina, Brasil. An Bras Dermatol. 2007;82:227-32.

27. Marsden Jr, Newton-Bishop JA, Burrows L, Cook M, Corrie PG, Cook M, et al. Revised U.K. guidelines for the management of cutaneous melanoma 2010. Brit J of Dermatol. 2010; 163:238-56.

28. Balch CM, Buzaid AC, Soong SJ, Atkins MB, Cascinelli N, Coit DG, et al. Final Version of the American Joint Committee on Cancer Staging System for Cutaneous Melanoma. J Clin Oncol. 2001;19:363548.

29. Lages RB, Barbosa PB, Almeida IP, Lopes LR, Filho LL. Deteç̧ão precoce do câncer de pele: experiência de campanha de prevenção no Piauí-Brasil. Ver Bras Promo Saúde. 2012;25:221-7.

30. Monzon J. CDKN2A mutations in multiple primary melanoma. N Engl J Med. 1998;338:879.

31. Sociedade Brasileira de Dermatologia. Análise de dados das campanhas de prevenção ao câncer da pele promovidas pela Sociedade Brasileira de Dermatologia de 1999 a 2005. An Bras Dermatol. 2006;81(6):533-9.24. Bazzocchi A, Ferrari F, Diano D, Albisinni U, Battista G, Rossi C, et al. Incidental Findings with DualEnergy X-Ray Absorptiometry: Spectrum of Possible Diagnoses. Calcified Tissue International. 2012; 91:149-156.

25. Hauache OM, Vieira JG, Alonso G, Martins LR, Brandao C. Increased hip bone mineral density in a woman with gluteal silicon implant. J Clin Densitom. 2000; 3:391-393.

26. Evans CE, Ward C, Braidman IP. Breast carcinomas synthesize factors which influence osteoblast-like cells independently of osteoclasts in vitro. J Endocrinol. 1991; 128:R5-R8.

27. Tolar J, Teitelbaum SL, Orchard PJ. Osteopetrosis. N Engl J Med. 2004; 351:2839-2849.

28. Taguchi M, Takeuchi Y. Anabolic effects of statin and beta-blocker on bone metabolism. Clin Calcium. 2004;14:89-94.

29. Polovina S, Popovic V, Duntas L, Milic N, Micic D. Frax score calculations in postmenopausal women with subclinical hypothyroidism. Hormones (Athens) 2013. 12:439-448. 